\title{
Estimation of Chronic Subdural Hematoma Size Using CT Imaging: A Comparison of In-Plane Thickness to 3D Volumetry
}

\author{
Milo Stanišić ${ }^{*}$, Inge Rasmuss Groote ${ }^{2}$, John Hald ${ }^{3}$, Are Hugo Pripp ${ }^{4}$ \\ ${ }^{1}$ Department of Neurosurgery, Oslo University Hospital, Oslo, Norway \\ ${ }^{2}$ Department of Psychology, Oslo University, Oslo, Norway \\ ${ }^{3}$ Department of Radiology, Oslo University Hospital, Oslo, Norway \\ ${ }^{4}$ Unit of Biostatistics and Epidemiology, Oslo University Hospital, Oslo, Norway \\ Email: "mstanisi@ous-hf.no
}

Received October 7, 2013; revised November 7, 2013; accepted November 14, 2013

Copyright (C) 2014 Milo Stanišić et al. This is an open access article distributed under the Creative Commons Attribution License, which permits unrestricted use, distribution, and reproduction in any medium, provided the original work is properly cited. In accordance of the Creative Commons Attribution License all Copyrights (C) 2014 are reserved for SCIRP and the owner of the intellectual property Milo Stanišić et al. All Copyright (C) 2014 are guarded by law and by SCIRP as a guardian.

\begin{abstract}
Backgrounds: Pre- and postoperative chronic subdural hematoma (CSDH) sizes have been used in clinical trials to predict the risk of postoperative recurrence. Commonly, dimensions of the pre- and postoperative lesions have been assessed by computerized tomography (CT) scans using maximum thickness as a linear measurement. Our goal was to characterize this common method for quantification of pre- and postoperative lesion sizes and to assess its estimation validity compared to estimation by hematoma volumetry. Methods: We prospectively investigated pre- and $1^{\text {st }}$ postoperative day CT scans of 107 adult surgical patients with uni- or bilateral CSDH. Preand postoperative thickness of CSDH was determined and then compared to pre- and postoperative lesion volume measured with 3D hematoma volumetry. Results: Pearson correlation coefficients between mean pre- and postoperative lesion thickness and mean pre- and postoperative lesion volume in the unilateral subgroup were 0.491 and 0.498 , respectively; in the bilateral subgroup 0.505 and 0.579 , respectively; and in the whole series 0.653 and 0.472, respectively. Conclusions: Pre- and postoperative thickness of CSDH does not offer reasonable approximations of the pre- and postoperative lesion size when compared with results from $3 \mathrm{D}$ volumetry in the unilateral subgroup, bilateral subgroup or overall.
\end{abstract}

\section{KEYWORDS}

\section{Chronic Subdural Hematoma; Computerized Tomography; Thickness; Volume}

\section{Introduction}

Although not adequately supported in the literature, large chronic subdural hematoma (CSDH) and substantial postoperative residual hematoma space are considered to be risk factors for developing postoperative recurrence [1-6]. Traditionally, dimensions of the pre- and postoperative lesions have been assessed by computerized tomography (CT) scans using maximum in-plane thickness as a linear measurement. This has been a standard method employed to estimate CSDH size [1,2,5,7-9]. Thus, over several decades, this linear estimate has been ex-

\footnotetext{
"Corresponding author.
}

tensively discussed throughout the literature as a risk factor for postoperative recurrence, but the findings have been contradictory [1-6,9-16]. Therefore, we hypothesized that approximation of CSDH size by linear measurement may be questionable. On the other hand, our recent study [16] showed that both large preoperative $\mathrm{CSDH}$ volume and large postoperative volume of residual hematoma space were radiological predictors of post-operative recurrence. Thus, current literature does not clearly define how to estimate and present neither the pre- nor the postoperative CSDH size. A study comparing $3 \mathrm{D}$ volumetric quantification of CSDH size and the linear measurement method has not yet been performed. 
Therefore, we conducted a prospective investigation in order to determine whether the thickness of CSDH could be used as a reasonable estimator of pre- and postoperative lesion size by comparing it with computer-assisted $3 \mathrm{D}$ volumetry estimation.

\section{Methods}

The study was approved by the Regional Ethical Committee of Health Region South-East Norway (S-06281a). Written and informed consent were obtained from patients or their close relatives prior to study inclusion.

\subsection{Patient Population and Management}

We prospectively investigated pre- and $1^{\text {st }}$ postoperative day CT scans, with and without contrast, of 107 adult patients with uni- or bilateral CSDHs who were surgically treated in the Department of Neurosurgery at Oslo University Hospital, during the time period January-December 2008. Hematoma evacuation was performed using a single burr-hole craniostomy and irrigation, followed by external, continuous closed-system drainage for a 24-hour period. The following radiological (CT scan) variables were analyzed: 1) site (unilateral or bilateral); 2) maximum in-plane thickness (pre- and postoperatively); 3) preoperative volume; and 4) postoperative residual cavity volume (i.e., the sum of residual rinsing fluid and residual air volumes in the hematoma cavity) following drain removal, on postoperative day one. Clinical data are not included because they are irrelevant to the subject matter of this study, which is lesion size quantification.

CSDHs were classified as unilateral or bilateral in site. The linear measurements, pre- and postoperative hematoma thickness, were estimated by measuring maximum in-plane thickness on each CT scan, at the level of CSDH; for unilateral CSDH the thickness of the one side was measured and for bilateral, the thicknesses of both sides were measured and the sum was determined $[5,7-9,16]$. Preoperative and postoperative CT scans were taken in order to estimate preoperative volume and residual cavity volume on postoperative day one, respectively, with the assistance of the software tool BrainVoyager QX 2.0. The method has been described previously [16]. In brief, images were electronically transferred in DICOM format to a workstation for image analysis. Preoperative CSDH and postoperative residual rinsing fluid in the hematoma cavity were delineated against soft tissues using the freehand selection tool on all tomographic images (slices), of each patient's CT scan. The area between the demarcation and the skull was filled using a seed-growing algorithm on each slice. Delineation of postoperative air content required no manual delineation due to excellent image contrast; and was performed using a manually placed starting point for each slice, and the same seed-growing algorithm as for the CSDH. Voxel dimensions from all individual slices and scans were extracted from the DICOM image header and used to calculate total preoperative CSDH metric volumes, postoperative rinsing fluid and air volumes. Computer-assisted 3D volumetric quantifications of preoperative volume and postoperative volume of residual cavity; one side in unilateral, summation of both sides in bilateral hematomas; were considered the standard.

\subsection{Statistical Analyses}

Data were analyzed using mean (standard deviation) or number of patients (percentage) for continuous or categorical variables, respectively. Statistical analyses were performed using the independent sample t-test, Pearson correlation or chi-square test, as appropriate. All statistical analyses were conducted using PASW Statistics 18 (IBM Corporation, Armonk, New York, USA) and considered statistically significant if $\mathrm{p}$-value $<0.05$.

\section{Results}

The sample consists of the same 107 patients with CSDH whose CT characteristics and their relationship to postoperative recurrence are all published in our previous study [16]. In brief, there were 84 (78.5\%) patients with unilateral and 23 (21.5\%) with bilateral hematomas; 72 males (67.3\%) and 35 females (32.7\%); the mean age of males was 71.4 (12.2) and of females 73.6 (13.9) years.

Statistics portraying preoperative linear measurement of hematoma size and hematoma volume, postoperative linear measurement of residual hematoma cavity size and volume of residual hematoma cavity; in relation to gender and site of lesion are presented in Tables 1 and 2, respectively. The correlations between the preoperative maximum thickness and the preoperative hematoma volume and between the postoperative maximum thickness of residual hematoma cavity and the postoperative residual hematoma cavity volume for the unilateral subgroup, bilateral subgroup and overall are shown in Table 3. Pearson correlation coefficients between mean preand postoperative lesion thickness and mean pre-and postoperative lesion volume in the unilateral subgroup were 0.491 and 0.498 , respectively; in the bilateral subgroup 0.505 and 0.579 , respectively; and in the whole series 0.653 and 0.472 , respectively.

As the colour-coded scatter-plot in Figure 1 shows, the preoperative maximum thickness of hematoma was an inaccurate estimator of the preoperative hematoma volume in an individual patient with unilateral or bilateral CSDHs. To give an example, an individual patient with unilateral CSDH who has a preoperative hematoma thickness of $27 \mathrm{~mm}$ may have the lower determined preoperative hematoma volume of $60 \mathrm{ml}$ or the upper vol- 
Table 1. Site of haematoma, pre- and postoperative haematoma thickness and pre- and postoperative haematoma volume in 107 patients with CSDH in relation to gender.

\begin{tabular}{|c|c|c|c|}
\hline CT features of CSDH & $\begin{array}{c}\text { Male } \\
\mathrm{N}=72(67.3 \%)\end{array}$ & $\begin{array}{c}\text { Female } \\
\mathrm{N}=35(32.7 \%)\end{array}$ & p-value \\
\hline unilateral site & $56(77.8 \%)$ & $28(80 \%)$ & \\
\hline bilateral site & $16(22.2 \%)$ & $7(20 \%)$ & 0.793 \\
\hline \multicolumn{4}{|l|}{ preoperative } \\
\hline maximum thickness of CSDH (SD) (mm) a & $31.5(11.7)$ & $26.4(8.1)$ & 0.011 \\
\hline volume of CSDH (SD) (ml) b & $167.9(75.8)$ & $127.1(49.4)$ & $<0.001$ \\
\hline \multicolumn{4}{|l|}{ Postoperative $^{*}$} \\
\hline maximum thickness of residual CSDH cavity (SD) (mm) c & $16.0(5.1)$ & $13.5(5.1)$ & 0.022 \\
\hline volume of residual CSDH cavity (SD) (ml) d & $120.3(65.5)$ & $83.7(38.3)$ & 0.123 \\
\hline
\end{tabular}

$\mathrm{N}=$ number of patients (percentage); Values are expressed as mean (standard deviation); a = preoperative maximum in-plane thickness in unilateral or sum of both sides in bilateral haematoma; $\mathrm{b}=$ preoperative volume in unilateral or sum of both sides in bilateral haematoma; $*=$ on $1^{\text {st }}$ postoperative day; $\mathrm{c}=$ postoperative maximum in-plane thickness of residual cavity in unilateral or sum of both sides in bilateral haematoma; $d=$ postoperative residual haematoma cavity volume (i.e., the sum of residual rinsing fluid and air volumes) in unilateral or sum of both sides in bilateral haematoma.

Table 2. Pre- and postoperative haematoma thickness and pre- and postoperative haematoma volume in 107 patients with CSDH in relation to site of lesion.

\begin{tabular}{|c|c|c|c|c|}
\hline CT features of CSDH & $\begin{array}{c}\text { All Patients } \\
\mathrm{N}=107(100 \%)\end{array}$ & $\begin{array}{c}\text { Unilateral } \\
\mathrm{N}=84(78.5 \%)\end{array}$ & $\begin{array}{c}\text { Bilateral } \\
\mathrm{N}=23(21.5 \%)\end{array}$ & p-value \\
\hline \multicolumn{5}{|l|}{ preoperative } \\
\hline volume of CSDH (SD) (ml) b & $154.8(70.8)$ & $136.5(50.2)$ & $221.1(93.4)$ & $<0.001$ \\
\hline \multicolumn{5}{|l|}{ postoperative $^{*}$} \\
\hline volume of residual CSDH cavity (SD) $(\mathrm{ml})^{\mathrm{d}}$ & $108.3(60.4)$ & $91.4(42.7)$ & $170.1(74.9)$ & $<0.001$ \\
\hline
\end{tabular}

$\mathrm{N}=$ number of patients (percentage); Values are expressed as mean (standard deviation); a = preoperative maximum in-plane thickness in unilateral or sum of both sides in bilateral haematoma; $\mathrm{b}=$ preoperative volume in unilateral or sum of both sides in bilateral haematoma; $*=$ on $1^{\text {st }}$ postoperative day; $\mathrm{c}=$ postoperative maximum in-plane thickness of residual cavity in unilateral or sum of both sides in bilateral haematoma; $d=$ postoperative residual haematoma cavity volume (i.e., the sum of residual rinsing fluid and air volumes) in unilateral or sum of both sides in bilateral haematoma.

Table 3. Correlation between pre- and postoperative haematoma thickness and pre- and postoperative haematoma volume in 84 patients with unilateral CSDH, 23 with bilateral CSDH and in the entire series.

\begin{tabular}{|c|c|c|c|}
\hline CT features of CSDH & $\begin{array}{c}\text { All Patients }(\mathrm{N}=107) \\
\text { r-value }\end{array}$ & $\begin{array}{c}\text { Unilateral }(\mathrm{N}=84) \\
\text { r-value }\end{array}$ & $\begin{array}{c}\text { Bilateral }(\mathrm{N}=23) \\
\text { r-value }\end{array}$ \\
\hline \multicolumn{4}{|l|}{ preoperative } \\
\hline volume of CSDH & 0.653 & 0.491 & 0.505 \\
\hline \multicolumn{4}{|l|}{ postoperative $^{*}$} \\
\hline volume of residual CSDH cavity & 0.472 & 0.498 & 0.579 \\
\hline
\end{tabular}

$\mathrm{N}=$ number of patients; $\mathrm{r}=$ Pearson correlation coefficient; ${ }^{*}=$ on $1^{\text {st }}$ postoperative day after drain removal.

ume of $225 \mathrm{ml}$; the difference is $165 \mathrm{ml}$ if a linear relationship is assumed. As another example, an individual patient with bilateral CSDH who has a preoperative hematoma thickness of $37 \mathrm{~mm}$ may have the lower determined preoperative hematoma volume of $75 \mathrm{ml}$ or the upper volume of $380 \mathrm{ml}$ (305 $\mathrm{ml}$ range).

It can be readily appreciated from Figure 2 that postoperative maximum thickness of residual hematoma cav- ity of an individual patient with unilateral or bilateral CSDHs was an inaccurate estimator of the postoperative lesion load, when compared to results from 3D volumetry. As an example, consider two patients with unilateral CSDH who had a $16 \mathrm{~mm}$ postoperative thickness of residual hematoma cavity; this could represent a $60 \mathrm{ml}$ postoperative volume of residual hematoma cavity in the one and a volume of $240 \mathrm{ml}$ in the other; the difference 


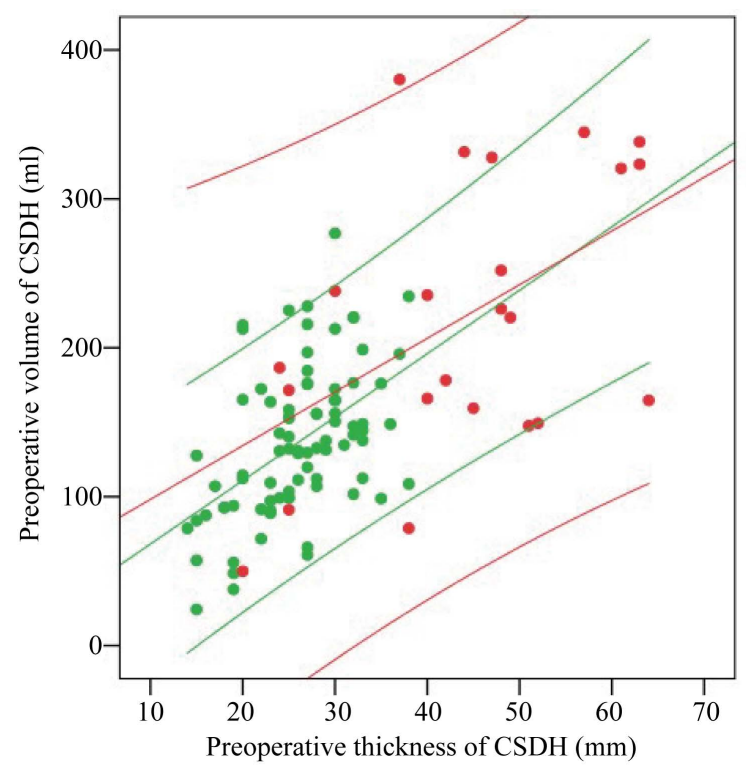

Figure 1. The relationship between the preoperative maximum in-plane thickness of hematoma and the preoperative hematoma volume of each individual patient in this series with individual 95\% CI. Green points = patients with unilateral CSDH. Red points = patients with bilateral CSDH. (See the explanation in the text).

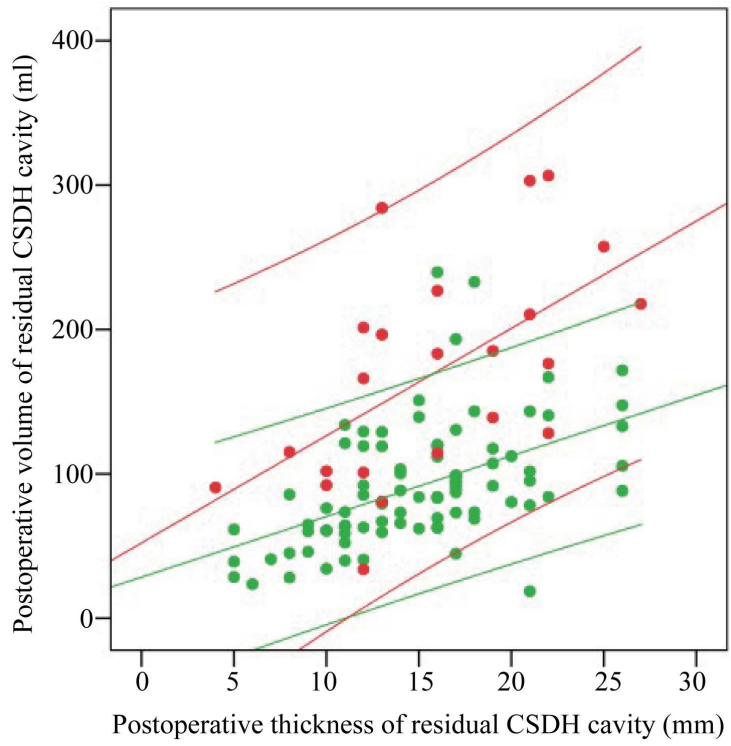

Figure 2. The relationship between the postoperative maximum in-plane thickness of residual hematoma cavity and the postoperative volume of residual hematoma cavity of each individual patient in this series with individual 95\% CI. Green points = patients with unilateral CSDH. Red points = patients with bilateral CSDH. (See the explanation in the text).

between them is then $180 \mathrm{ml}$ if a linear relationship is assumed. Similarly, Figure 2 shows that an individual patient with bilateral CSDH who has a $22 \mathrm{~mm}$ postoperative thickness of residual cavity could have the lower determined postoperative volume of residual hematoma cavity of $130 \mathrm{ml}$ or the upper volume of $308 \mathrm{ml}(178 \mathrm{ml}$ range).

\section{Discussion}

Preoperative hematoma thickness is considered to be an approximate, but reasonable indicator of the hematoma size and brain compression. Similarly, postoperative thickness is considered to be an approximate, but reasonable indicator of postoperative residual hematoma space size and postoperative brain decompression. Large preoperative hematoma thickness has repeatedly been reported to be associated with an increased risk of recurrence [1,2, 13,15], corroborating a general opinion that a large CSDH is a risk factor for relapse; on the contrary, it has also repeatedly been reported that preoperative hematoma thickness is not associated with postoperative recurrence rate $[4,5,9,11,12,14,16]$. Similarly, it has been reported that large postoperative thickness of residual hematoma cavity is associated with an increased risk of recurrence [1], corroborating a general opinion that persistence of residual space postoperatively may predispose to recurrence; on the contrary again, it has been reported that this radiological variable is not associated with recurrence rate $[9,16]$. Given the contradictory nature of the aforementioned findings, it seems difficult to determine whether pre- and postoperative thickness is valid estimator of lesion load that should play any prognostic role in individual cases. Questions should be raised as to why the findings of previous studies have been conflicting and to what use we have of these controversial findings.

By virtue of a CSDH being an "old" circumscribed and liquefied collection of blood/ blood breakdown products encapsulated between the inner layer of the dura and the external surface of the brain at the arachnoid membrane, a CSDH can be sickle shaped and this is the basis for the linear measurement which is taken to approximate its size. However, the sickle shape of CSDH may vary considerably, depending on whether the lesion is located laterally over the hemisphere, if it extends to the cranial base, to the vertex or if it is interhemispheric. Furthermore, as Sucu et al. [17] have emphasized, axial CT slices above the superior temporal line are no longer perpendicular to the cranium or $\mathrm{CSDH}$, instead they run virtually tangential to the hematoma because of the curvature of the cranial vault; therefore, the thickness of the hematoma measured on a slice close to vertex appears to be thicker than it actually is. Additionally, it is important to note that the method of measuring hematoma thickness is not standardized and varies between studies; in some studies thickness was measured on the CT scan at the level of its maximum in-plane thickness [5,7-9,16] and in others at the level of maximum in-plane thickness, at the level of lateral ventricles $[1,2]$. Therefore, we hy- 
pothesized in this study that approximation of CSDH size by linear measurement is inexact and further that expressing lesion size in volumetric units could reduce the effect of approximations.

In this study, we found neither good correlations between the preoperative maximum thickness and the preoperative hematoma volume, nor between the postoperative maximum thickness and the postoperative volume of residual hematoma cavity in unilateral $\mathrm{CSDH}$, bilateral CSDHs and in the entire series (Table 3). Judging from the scattered results shown in Figures 1 and 2, the preand postoperative thickness of individual lesions in patients with unilateral or bilateral hematoma seem to be inaccurate estimators of the lesion volume when compared to 3D volumetry estimations; consequently, it seems that the pre- and postoperative hematoma thickness are not reliable estimators of pre- or postoperative lesion sizes.

In this study, we used computer-assisted volumetry derived from CT imaging, and found it to be quite an accurate method for quantification of preoperative hematoma size and postoperative size of residual hematoma cavity by virtue of the fact that it takes into consideration irregular lesion shape, different lesion density appearances and also includes each voxel in which the lesion appears. Additionally, the pre- and postoperative CSDH volume seems to be a more reasonable indicator of the preoperative brain compression and postoperative decompression than thickness. The results of this study reveal that quantification of pre- and postoperative lesion sizes based on computer-assisted 3D volumetry are inconsistent with quantification of sizes using linear measurements.

Currently, computer-assisted CSDH volumetry requires manual segmentation because no dedicated edgedetection software solutions exist for reliable delineation of the soft tissue boundaries created by $\mathrm{CSDH}$, which is necessary in order to provide fully automated lesion volumetry. However, numerous disseminated software solutions are in existence and allow for straightforward semi-automatic volumetric assessments, as described in the current study. The extra time spent using 3D volumetry versus linear in-plane measurement is approximately 10 minutes per study; so, for merely 10 extra minutes, measurement that represents lesion size more accurately can be obtained.

Based on this prospective, single centre study including 107 patients with unilateral CSDH and bilateral CSDHs, it seems that pre- and postoperative thickness of CSDH do not offer reasonable approximations of the preand postoperative lesion size when compared to results from 3D volumetry in the unilateral subgroup, bilateral subgroup or in the entire series. Further, we speculate that volume should routinely be used as an estimator of pre- and postoperative lesion sizes in the determination of risk for postoperative recurrence. It seems that the linear measure of lesion size is outdated method for use for this purpose on account of the lack of their ability to assess lesion size correctly and predict postoperative recurrence consistently.

\section{Conclusion}

Pre- and postoperative lesion volumes were powerful estimators of the preoperative hematoma size and the postoperative size of residual CSDH cavity. Pre- and postoperative maximum in-plane thickness of hematoma does not offer reasonable approximations of the pre- and postoperative lesion size. The results of this study may help identify useful radiological tools that could be of assistance in both the optimal clinical decision-making and the determination of risk of postoperative CSDH recurrence.

\section{Acknowledgments}

This study was supported by a grant from the Department of Neurosurgery, Oslo University Hospital, Oslo, Norway.

\section{Conflict of Interest}

We declare no conflict of interest.

\section{REFERENCES}

[1] M. E. Baé, H. Wessling, H. C. Salca and P. H. Echeverría, "Use of Twist-Drill Craniostomy with Drain in Evacuation of Chronic Subdural Hematomas: Independent Predictors of Recurrence," Acta Neurochirurgica, Vol. 153, No. 5, 2011, pp. 1097-1103.

[2] K. H. Chon, J. M. Lee, E. J. Koh and H. Y. Choi, "Independent Predictors for Recurrence of Chronic Subdural Hematoma," Acta Neurochirurgica, Vol. 154, No. 9, 2012, pp. 1541-1548.

[3] H. El-Kadi, V. J. Miele and H. H. Kaufman, "Prognosis of Chronic Subdural Hematomas,” Neurosurgery Clinics of North America, Vol. 11, No. 3, 2000, pp. 553-567.

[4] K. Mori and M. Maeda, "Surgical Treatment of Chronic Subdural Hematoma in 500 Consecutive Cases: Clinical Characteristics, Surgical Outcome, Complications, and Recurrence Rate,” Neurologia Medico-Chirurgica (Tokyo), Vol. 41, No. 8, 2001, pp. 371-381.

http://dx.doi.org/10.2176/nmc.41.371

[5] H. Nakaguchi, T. Tanishima and N. Yoshimasu, "Relationship between Drainage Catheter Location and Postoperative Recurrence of Chronic Subdural Hamatoma after Burr-Hole Irrigation and Closed-System Drainage,” Journal of Neurosurgery, Vol. 93, No. 5, 2000, pp. 791-795. http://dx.doi.org/10.3171/jns.2000.93.5.0791

[6] K. Torihashi, N. Sadamasa, K. Yoshida, O. Narumi, M. Chin and S. Yamagata, "Independent Predictors for Re- 
currence of Chronic Subdural Hematoma: A Revive of 343 Consecutive Surgical Cases,” Neurosurgery, Vol. 63, No. 6, 2008, pp. 1125-1129.

[7] Y. Okada, T. Akai, K. Okamoto, T. Iida, H. Takata and H. Iizuka, "A Comparative Study of the Treatment of Chronic Subdural Hematoma-Burr Hole Drainage versus Burr Hole Irrigation,” Surgical Neurology, Vol. 57, No. 6, 2002, pp. 405-409. http://dx.doi.org/10.1016/S0090-3019(02)00720-6

[8] C. Smely, A. Madlinger and R. Scheremet, "Chronic Subdural Hematoma-A Comparison of Two Different Treatment Modalities,” Acta Neurochirurgica (Wien), Vol. 139, No. 9, 1997, pp. 818-826.

http://dx.doi.org/10.1007/BF01411399

[9] M. Stanisic, M. Lund-Johansen and R. Mahesparan, “Treatment of Chronic Subdural Hematoma by Burr-Hole Craniostomy in Adults: Influence of Some Factors on Postoperative Recurrence,” Acta Neurochirurgica (Wien), Vol. 147, No. 12, 2005, pp. 1249-1257.

[10] T. Sundstrøm, C. A. Helland, M. Aarhus and K. Wester, "What Is the Pressure in Chronic Subdural Hematomas? A Prospective, Population-Based Study,” Journal of Neurotrauma, Vol. 29, No. 1, 2012, pp. 137-142.

[11] A. Frati, M. Salvati, F. Mainiero, F. Ippoliti, G. Rocchi, A. Roco, E. Caroli, G. Cantore and R. Delfini, "Inflammation Markers and Risk Factors for Recurrence in $35 \mathrm{~Pa}-$ tients with a Posttraumatic Chronic Subdural Hematoma: A Prospective Study,” Journal of Neurosurgery, Vol. 100, No. 1, 2004, pp. 24-32. http://dx.doi.org/10.3171/jns.2004.100.1.0024

[12] A. R. Kristof, M. J. Grimm and B. Stoffel-Wagner, “Cerebrospinal Fluid Leakage into the Subdural Space: Poss- ible Influence on the Pathogenesis and Recurrence Frequency of Chronic Subdural Hematoma and Subdural Hygroma,” Journal of Neurosurgery, Vol. 108, No. 2, 2008, pp. 275-280.

[13] K. Matsumoto, K. Akagi, M. Abekura, H. Ryujin, M. Ohkawa, N. Iwasa and C. Akiyama, "Recurrence Factors for Chronic Subdural Hematomas after Burr-Hole Craniostomy and Closed System Drainage,” Neurological Research, Vol. 21, No. 3, 1999, pp. 277-280.

[14] P. Taussky, J. Fandino and H. Landolt, "Number of Burr Holes as Independent Predictor of Postoperative Recurrence in Chronic Subdural Haematoma,” British Journal of Neurosurgery, Vol. 22, No. 2, 2008, pp. 279-282.

[15] H. Yamamoto, Y. Hirashima, H. Hamada, N. Hayashi, H. Origasa and S. Endo, "Independent Predictors of Recurrence of Chronic Subdural Hematoma: Results of Multivariate Analysis Performed Using a Logistic Regression Model,” Journal of Neurosurgery, Vol. 98, No. 6, 2003, pp. 1217-1221. http://dx.doi.org/10.3171/jns.2003.98.6.1217

[16] M. Stanišić, J. Hald, I. A. Rasmussen, A. H. Pripp, J. Ivanović, F. Kolstad, J. Sundseth, M. Züchner and K. F. Lindegaard, "Volume and Densities of Chronic Subdural Haematoma Obtained from CT Imaging as Predictors of Postoperative Recurrence: A Prospective Study of 107 Operated Patients,” Acta Neurochirurgica, Vol. 155, No. 2, 2013, pp. 323-333.

[17] H. K. Sucu, M. Gokmen and F Gelal, "The Value of XYZ/2 Technique Compared with Computer-Assisted Volumetric Analysis to Estimate the Volume of Chronic Subdural Hematoma,” Stroke, Vol. 36, No. 5, 2005, pp. 998-1000. 